\title{
A qualidade da informação para a tomada de decisão sob a perspectiva do sensemaking: uma ampliação do campo
}

\author{
Gustavo Abib \\ Doutor em administração pela Universidade Federal do \\ Rio Grande do Sul, Porto Alegre, RS - Brasil. Professor \\ assistente da Universidade Federal do Paraná, Departamento \\ de Administração - Curitiba, PR - Brasil \\ E-mail: gustavo.abib@gmail.com
}

\section{Resumo}

Este ensaio teórico busca, sob a ótica do sensemaking proposto por Weick (1995), analisar a qualidade da informação no processo decisório organizacional, sugerindo uma proposta para ampliar as técnicas utilizadas para mensurar a qualidade da informação. Para tanto, conceitua informação e processo decisório; aborda os conceitos do sensemaking e discute as dimensões da mensuração da qualidade da informação - apresentando os relacionamentos entre estas dimensões e as propriedades do sensemaking propostas por Weick. Finalmente, o ensaio sugere-se uma agenda baseada na abordagem proposta para utilização em pesquisas empíricas e para a análise em profundidade da aplicação deste relacionamento.

Palavras-chave

Qualidade da informação. Tomada de decisão. Sensemaking.

The information quality for decision-making in the sensemaking perspective: an extension of the field

\begin{abstract}
The objective of this paper, from the sensemaking perspective proposed by Weick (1995), to analyze the information quality used in the organizational decision-making process, suggesting a proposal to expand the techniques used to measure the quality of information. Therefore, this paper: presents the concept of information and decision-making process; addresses the concepts of sensemaking and discusses the dimensions of measuring the quality of information - presenting the relationships between these dimensions and properties of sensemaking proposed by Weick. Finally, this paper suggests an agenda based on the approach proposed for use in empirical research and for indepth analysis of the application of this relationship.
\end{abstract}

\section{Keywords}

Information quality. Decision-making. Sensemaking.

Ci. Inf., Brasília, DF, v. 39 n. 3, p.73-82, set./dez., 2010

\section{INTRODUÇÃO}

Embora a informação não seja considerada uma novidade no campo de pesquisa, pois já é investigada há muito tempo, seu foco de estudo pode ser considerado bem amplo. A qualidade da informação é um dos tópicos sobre informação que tem originado vários estudos internacionais (EVEN e SHANKARANARAYANAN, 2007; PIPINO, LEE e YANG, 2002; WAND e WANG 1996; WANG et. al. 1995) e nacionais (OLETO, 2006; NEHMY e PAIM, 1998).

Inicialmente, é importante conceituar a informação. O conceito sobre informação e seus sistemas vem, aos poucos, sendo ampliado com o avanço das pesquisas. Primariamente definia-se informação como o resultado da análise de dados que, por sua vez, eram considerados quaisquer elementos identificados em sua forma bruta, que por si sós não conduzem a uma compreensão de determinado fato ou situação, conforme apontam Laudon e Laudon (1996).

Outra definição de informação é apresentada por Masuda (1982, p. 74), que determina: “a informação é uma relação situacional, observada entre sujeito e objeto, que torna possível a seleção da ação pela qual o próprio sujeito pode atingir alguma espécie de valor de uso". Ciborra (1992) afirma que a informação pode ser compartilhada somente se, em ambas as pontas, o mesmo entendimento do contexto relevante estiver disponível e se os atores presentes na troca de informações entender os sentido dos sinais transmitidos e recebidos, fazendo referência a contextos similares de interpretação. Percebe-se, na literatura, uma dificuldade em conceituar a informação, pois ela pode ter muitos significados. Nota-se, obviamente, que ela sempre está submetida a um processo de interpretação. 
Rotineiramente, utiliza-se a informação no processo de tomada de decisões (BAZERMAN, 2004; HAMMOND, KEENEY e RAIFFA, 2004; CLEMEN, 1996; Mcgee e PRUSAK, 1994). Para Clemen (1996), o processo decisório muitas vezes conta com um conjunto incompleto de informações que servem de subsídio ao processo decisório. Neste sentido, consciente da imperfeição das informações disponíveis, o agente decisor deve se utilizar de informações com qualidade para minimizar os efeitos de uma escolha prejudicial.

Daft e Weick (2005) destacam que a tomada de decisão geralmente parte dos processos de informação e interpretação nas organizações, e que as decisões estão associadas aos diversos modos de interpretação. Em muitas organizações, o processo de tomada de decisões constitui importante aspecto da atividade de gerenciamento. Espera-se que os gestores tomem melhores decisões e que estas resultem em ações produtivas, rapidez na resolução de problemas e melhor desempenho organizacional (JUNG, 2004). Para esse autor, o processo de tomada de decisão pelos indivíduos nas organizações não representa uma tarefa fácil, particularmente quando é analisado o problema da complexidade, da quantidade e da qualidade da informação empregada no processo decisório.

Vários estudos (BELKIN, 1982; WILSON, 2000; KUHLTHAU, 2004; entre outros) colocaram o ator social como foco na interpretação da necessidade de informação (análise do processo de coleta e uso das informações). Belkin (1982) analisou a necessidade de informações por parte dos usuários. Já Kuhlthau (2004) identificou "zonas de intervenção", classificando três delas como "identificadora", "assessor" e "conselheiro". Em todos esses estudos a análise perpassa por questões sociais, contextuais e pessoais de valoração da informação.

Percebe-se que um importante atributo da informação para a tomada de decisão é a qualidade. Para Wand e Wang (1996), a qualidade da informação é apresentada como um conceito multidimensional.
Pesquisas examinaram diferentes perspectivas para o gerenciamento da qualidade da informação: analítica; técnica; operacional e gerencial (Wang et al., 1995), porém percebe-se: na literatura a existência de uma gama de atributos divergentes para conceituar a qualidade da informação. Neste sentido, questionase: por que existe dificuldade para se mensurar a qualidade da informação?

Para responder à questão anterior, a perspectiva cognitiva dá um passo em direção à compreensão subjetiva da informação (CAPURRO, 2007). Esse autor afirma que as pessoas têm diferentes bagagens educacionais e desempenham diferentes funções na divisão do trabalho e na sociedade, valorando de forma diversa as informações.

Even e Shankaranarayanan (2007) destacam que os atuais métodos de mensuração da qualidade da informação estão classificados em fatores estruturais e técnicos, permitindo uma mensuração imparcial da qualidade, não se preocupando com os fatores contextuais e pessoais. Este ensaio teórico busca aproximar a abordagem do sensemaking, proposta por Weick (1995), para a mensuração da qualidade da informação a ser utilizada para a tomada de decisão. Desse modo, tal aproximação busca extrapolar os fatores puramente técnicos e atribuir importância também aos fatores pessoais envolvidos no processo. Para tanto, este ensaio faz uma breve revisão teórica sobre o conceito de sensemaking, apresentando em seguida a qualidade da informação e seus relacionamentos com estes conceitos. Por fim, sugere-se uma agenda de pesquisa para ampliação do tema.

\section{SENSEMAKING SEGUNDO WEICK (1995): A CRIAÇÃO DE SENTIDO}

As organizações e os indivíduos tentam descobrir uma resposta aceitável antes que seus recursos se esgotem, que os concorrentes monopolizem o mercado, que o interesse das pessoas mude ou que as oportunidades em outros setores ambientais dominem 
A qualidade da informação para a tomada de decisão sob a perspectiva do sensemaking: uma ampliação do campo

essa busca (DAFT e WEICK, 2005). Para esses autores, todas estas atividades representam uma forma de interpretação que requer que as pessoas que compõem as organizações necessitem tomar decisões.

Pode-se pensar sobre o mundo de diferentes formas, relacionando os conceitos às experiências pessoais vivenciadas nesse mundo e, a partir daí, formar julgamentos que podem afetar as intenções e também as ações (CHECKLAND, 1998). Cada ser humano traz consigo a capacidade de, seletivamente, perceber e interpretar o ambiente onde vive. Uma das características marcantes do ser humano é a sua capacidade para o diálogo, a qual possibilita debates e discussões que afetam as percepções, os julgamentos, as intenções e as ações.

As pessoas, normalmente, tentam interpretar as próprias ações; definir o próprio aprendizado; e resolver o problema do que deve ser feito em seguida. A construção da interpretação acerca do ambiente é uma necessidade básica dos indivíduos e das organizações. O processo de construção da interpretação pode ser influenciado pela natureza da resposta procurada, pelas características do ambiente, pela experiência prévia do questionador e pelo método usado para adquiri-la (DAFT e WEICK, 2005; DERVIN, 1998).

A criação de significado na literatura, de acordo com Choo (2003), tem sido definida de várias maneiras por diferentes pesquisadores. March e Olsen (1976) analisaram a criação de significado como parte do aprendizado experimental, no qual "indivíduo e organização dão significado à sua experiência e modificam seu comportamento de acordo com suas interpretações". Starbuck e Milliken (1988) observaram que "a criação de significado tem muitos aspectos distintos - no mínimo, compreender, explicar, atribuir, extrapolar e prever [...]. O que esses processos têm em comum é que eles implicam colocar estímulos dentro de estruturas (ou esquemas) que lhes dêem sentido".

Klein e Myers (1999) destacam que o interpretativismo pode auxiliar os pesquisadores de sistemas de informações a entender o pensamento humano e a ação social no contexto da organização. Isso tem o potencial para produzir profundos entendimentos sobre os fenômenos dos sistemas de informações, incluindo o gerenciamento e o desenvolvimento desses sistemas e questões relacionadas à informação.

O conceito de sensemaking para Weick (1995) significa produzir sentido. Pode ser analisado também sob a ótica da estruturação do desconhecido (WATERMAN, 1990 apud WEICK, 1995). Figurativamente, o sensemaking é um processo espiralado que compreende uma série de eventos ocorridos através do tempo. Para explicitar melhor o que compreende o sensemaking, WEICK (1995) compara-o com uma atividade ou processo, diferindo da simples interpretação. Para esse autor, o sensemaking é o ato de construir, filtrar, referenciar, criar factibilidade e transformar em tangível algo que antes era subjetivo.

Outros conceitos de sensemaking inseridos na obra de Weick (1995) são encontrados, tais como uma busca interativa de informação; a atribuição de significados e ação; os mecanismos que os indivíduos utilizam para atribuir significado a eventos - incluindo padrões e regras para perceber, interpretar, crer e agir sob um contexto cultural; e um processo interpretativo necessário para que os indivíduos entendam e compartilhem suas compreensões. Choo (2003) complementa afirmando que esse processo de criação de sentidos é contínuo e que os indivíduos observam fatos passados, recontam pedaços da experiência e selecionam alguns pontos de referência para construir o significado.

De acordo com Devin (1998), o sensemaking tem sido desenvolvido por mais de 25 anos nos limites do discurso de comunidades. Estes incluíam inicialmente o campo da biblioteconomia e da ciência da informação (em que os estudos focavam a necessidade da informação e o alinhamento entre a informação e os sistemas). Busca-se atualmente uma expansão do campo utilizando-se do sensemaking como uma lente para analisar a qualidade da informação. 
O sensemaking sob a ótica de Dervin (1983) e Weick, Sutcliffe e Obstfeld (2005) apresenta uma realidade que não é completa, muito menos constante, de acordo com a afirmação de Clemen (1996). $\mathrm{Na}$ verdade essa realidade é vista como difusa, complexa e mutável. Neste sentido, o sensemaking surge como uma alternativa para compreender os processos de valoração (qualidade) da informação.

Diante de uma situação de incerteza, como no caso da tomada de decisões, os decisores procuram compreender o problema buscando coletar e interpretar informações construindo sentido através delas. Essa busca pela compreensão do processo de sensemaking é essencial para a diminuição da complexidade da decisão. Como as decisões envolvem contextos, problemas e atores diferentes, o processo do sensemaking está sempre presente (WEICK, SUTCLIFFE E OBSTFELD, 2005).

Weick (1995) identifica sete propriedades que fazem da criação de significado um processo individual. A criação de significado é vista como um processo:

a) fundado na construção da identidade;

b) retrospectivo;

c) que representa ambientes sensíveis;

d) social;

e) contínuo;

f) focado em e por pistas extraídas;

g) governado mais pela plausibilidade do que pela precisão;

Com base nas sete propriedades fundamentais do sensemaking procura-se, a seguir, estabelecer algumas relações entre o processo de sensemaking e a qualidade da informação para tomada de decisão nas organizações.

\section{SENSEMAKING E A QUALIDADE DA INFORMAÇÃO PARA TOMADA DE DECISÃO}

$\mathrm{Na}$ literatura de sistemas de informações, a qualidade da informação é uma dimensão utilizada para avaliar o sucesso desses sistemas, sendo fundamental para a qualidade da decisão (EVEN e SHANKARANARAYANAN, 2007; JUNG, 2004). Percebe-se, nos estudos realizados, grande variedade de formas e atributos para conceituar a qualidade da informação - fato este que retrata a dificuldade desta mensuração.

A qualidade, quando tratada isoladamente, constitui um substantivo abstrato, um construto de fácil entendimento por meio do senso comum, mas de complexo entendimento quando se busca uma definição mais rigorosa e quando, durante a construção de uma teoria, procura-se relacioná-lo com outras variáveis (OLETO, 2006).

Autores como Wand e Wang (1996) comparam a qualidade da informação à qualidade do produto - que, por sua vez, depende da maneira pela qual é desenhado e produzido, não levando em consideração o contexto no qual ele é produzido e utilizado, nem tampouco os usuários. No estudo de Even e Shankaranarayanan (2007), esses autores ressaltam a mensuração incompleta da qualidade da informação, uma vez que a maioria dos estudos não leva em consideração fatores pessoais e contextuais.

Assim, a qualidade da informação precisa ser definida utilizando-se múltiplas dimensões que possibilitem melhor mensuração da qualidade. Diferentes métricas têm sido propostas para mensurar, de maneira quantitativa, a qualidade da informação (REDMAN, 1986; PIPINO et al., 2002).

A análise da qualidade da informação nas empresas deve passar pela observação das percepções subjetivas dos indivíduos envolvidos com a informação (PIPINO, LEE e YANG, 2002). A análise da qualidade proposta por esses autores leva em consideração as percepções subjetivas dos 
A qualidade da informação para a tomada de decisão sob a perspectiva do sensemaking: uma ampliação do campo

indivíduos e o contexto no qual tanto as informações quanto os indivíduos estão inseridos. Para mensurar a qualidade da informação, esses autores propõem que ela seja analisada sob a ótica de 16 dimensões, como mostra o quadro 1 :

Considerando a base filosófica do sensemaking como foco no ator individual e a visão de Dervin (1998) como a informação sendo o produto de uma construção subjetiva, de posse dos conceitos do sensemaking e dos atributos sobre a qualidade da informação proposta por Pipino, Lee e Yang (2002), é tecida uma aproximação destes conceitos nos tópicos a seguir:

\section{QUADRO 1}

\section{Dimensões da qualidade da informação}

\begin{tabular}{|c|c|}
\hline DIMENSÕES & DEFINIÇÕES \\
\hline Acessibilidade & $\begin{array}{l}\text { Em qual extensão a informação está disponível, ou quão facilmente e } \\
\text { rapidamente é coletada. }\end{array}$ \\
\hline $\begin{array}{l}\text { Quantidade de } \\
\text { Informação }\end{array}$ & $\begin{array}{l}\text { Em qual extensão o volume de informações é apropriado para a resolução do } \\
\text { problema ou para a tomada de decisão. }\end{array}$ \\
\hline Veracidade & Em qual extensão a informação é considerada verdadeira e digna de crédito. \\
\hline Integral - Completa & Em qual extensão a informação não está incompleta para a tomada de decisão. \\
\hline Concisão & A extensão pela qual a informação é condensadamente apresentada. \\
\hline Consistência & A extensão pela qual a informação é apresentada no mesmo formato. \\
\hline $\begin{array}{l}\text { Facilidade de } \\
\text { Manipulação }\end{array}$ & $\begin{array}{l}\text { A extensão pela qual a informação pode ser manipulada e utilizada em } \\
\text { diferentes tarefas. }\end{array}$ \\
\hline Livre de erros & A extensão pela qual a informação é correta e confiável. \\
\hline Interpretabilidade & $\begin{array}{l}\text { A extensão pela qual a informação está apropriada de linguagens, símbolos, } \\
\text { unidades e clareza de definições. }\end{array}$ \\
\hline Objetividade & Imparcialidade, não prejudicada e livre de tendências. \\
\hline Relevância & A extensão pela qual a informação é aplicável e útil para a tarefa em questão. \\
\hline Reputação & $\begin{array}{l}\text { A extensão pela qual a informação é altamente confiável em termos de fonte ou } \\
\text { conteúdo. }\end{array}$ \\
\hline Segurança & $\begin{array}{l}\text { A extensão na qual o acesso a informação é restrito com o intuito de manter a } \\
\text { segurança. }\end{array}$ \\
\hline Atual & $\begin{array}{l}\text { A extensão na qual a informação é suficientemente atualizada para a tarefa em } \\
\text { questão. }\end{array}$ \\
\hline $\begin{array}{l}\text { Facilidade de } \\
\text { entendimento }\end{array}$ & A extensão na qual a informação é facilmente compreendida. \\
\hline Adição de valor & A extensão na qual a informação é benéfica e fornece vantagens com seu uso. \\
\hline
\end{tabular}

Fonte: Adaptado de Pipino, Lee e Yang (2002, p. 212)

Ci. Inf., Brasília, DF, v. 39 n. 3, p.73-82, set./dez., 2010 
Tome-se, por exemplo, um executivo que muda de uma empresa para outra que atua em um ramo diferente. Mesmo que as práticas de gestão sejam as mesmas, a construção da nova identidade é o que determinará a construção do sentido e a priorização das informações necessárias ao processo de tomada de decisão. Esse processo permitirá ao executivo identificar, analisar e julgar a integralidade das informações utilizadas na tomada de decisão. Como a formação da identidade é um processo, com o passar do tempo, desenvolve-se a facilidade de entendimento e interpretabilidade das informações.

Portanto, as dimensões mais relacionadas com esta propriedade são a integralidade da informação, a facilidade de entendimento e a interpretabilidade. A criação da identidade instrumentaliza os decisores a compreenderem as linguagens, os símbolos e unidades presentes no processo.

Depreende-se da análise que a construção da identidade auxilia o tomador de decisões a superar as barreiras estruturais (relacionadas aos processos sociais - eficiência) e também institucionais (relacionadas ao monitoramento do ambiente no qual a empresa se encontra).

\section{b) Retrospectivo}

A criação de sentido é retrospectiva, ou seja, trabalha com fatos que já ocorreram. Esta ideia, de acordo com Weick (1995), deriva dos trabalhos apresentados por Schultz's (1967 apud Weick, 1995), que tratava da "experiência significativa vivida". O ponto importante do sensemaking retrospectivo para Weick (1995) é a atividade na qual muitos possíveis significados podem ser sintetizados.

Em relação à qualidade da informação, uma dimensão que se encaixa nessa propriedade é a quantidade de informação. Existe um ponto ótimo em relação à quantidade, pois as pessoas não necessitam de mais informações quando precisam decidir. Em vez disso, elas necessitam de valores, prioridades e esclarecimentos sobre suas preferências. Outra dimensão tratada aqui é a reputação, que aborda a extensão pela qual a informação é confiável em relação à fonte e ao conteúdo. Neste momento, a análise retrospectiva traz a resposta, pois gera uma reflexão sobre o passado para a ação no presente. Por fim, outra dimensão que está relacionada é a objetividade, que pode ser influenciada negativamente - dependendo das experiências prévias do indivíduo. Capurro (2007) complementa afirmando que a visão através do domínio analítico está relacionada a uma visão hermenêutica, pois a valoração é determinada por um pré-entendimento do observador, alinhado aos conceitos do sensemaking.

c) Que representa ambientes sensíveis

Os indivíduos, na sua vida organizacional, produzem parte do ambiente no qual estão inseridos. A ideia de representação incita maior atenção para as atividades interdependentes, para os processos e para a mudança contínua.

Qualquer processo de obtenção e entendimento da informação é baseado no prévio desenvolvimento de um conhecimento sobre a situação. Tal estrutura cognitiva foi estudada na teoria organizacional por Weick (1979). Dado que Weick (1995) também sugere que o sensemaking pode se tornar crescentemente demarcado, à medida que as organizações se tornam mais maduras, também se pode esperar que o tempo desempenhe um papel importante nesse inter-relacionamento (WILSON, JARZABKOWSKI, 2004).

Quando se aborda o ambiente, busca-se compreender o contexto no qual a qualidade da informação é percebida. Para Pozzebon e Pinsonneault (2000), o estudo do contexto, ou da contextualidade da interação, é visto como inerente na investigação da reprodução social e envolve a dimensão de tempo-espaço e a presença dos atores, entre outros elementos. A influência da análise do contexto repousa na percepção de importância ou relevância da informação para a tomada de decisão de acordo com o momento vivido pelo gestor (situações contextuais). Tal 
A qualidade da informação para a tomada de decisão sob a perspectiva do sensemaking: uma ampliação do campo

propriedade está intimamente ligada às dimensões de relevância e adição de valor. A relevância é a extensão pela qual a informação é aplicável e útil para a tarefa em questão, sendo um processo no qual a relevância é obtida quando o indivíduo analisa o ambiente e o contexto no qual está inserido. Uma vez analisada a relevância, a dimensão de adição de valor é uma dimensão complementar da primeira.

\section{d) Social}

Sensemaking para Weick (1995) é um processo social (coletivo) e nunca solitário, porque o que a pessoa faz internamente é contingente nos outros. Para esse autor, as pessoas que estudam o sensemaking focam atenções nas conversas, discursos e diálogos, pois é através dessas formas de comunicação que o contato social é mediado.

$\mathrm{Na}$ análise da qualidade da informação, as dimensões de acessibilidade e segurança se encaixam nesta propriedade. A acessibilidade da informação para a tomada de decisão pode ser formal (através de sistemas) ou informal (por meio de pessoas), como observado por Beal (2004). Hammond, Keeney e Haiffa (2004) salientam que os tomadores de decisões preferem informações coletadas pelo contato social àquelas provenientes somente dos sistemas de gestão. Ciborra (1992) complementou tal ideia, afirmando que a informação pode ser dividida somente se, em ambas as partes, o mesmo entendimento do contexto relevante for obtido pelos atores, entendimento esse ocorrido por interações sociais e interpretativas. Do mesmo modo, a segurança da informação está intimamente ligada às pessoas que têm acesso a ela. Ocorrerá a segurança, se as pessoas compartilharem o mesmo entendimento sobre a importância da informação em questão.

\section{e) Processo contínuo}

A criação de significado é um processo contínuo, ou seja, não possui um início ou um fim definido. A ação da tomada de decisão nas organizações também pode ser considerada um fluxo (EISENHARDT e ZBARACKI, 1992; COHEN, MARCH e OLSEN, 1972), pois os indivíduos constantemente deparam-se com problemas, escolhas e soluções. Adicionalmente, o processo de criação de significado também é nãolinear.

Quando se analisaram os ambientes sensíveis, foram exploradas a dinamicidade dos contextos e sua importância para a qualidade da informação. Resultado desta dinamicidade, surge um processo contínuo de coleta, tratamento e uso da informação, convergindo para a atualização das informações. A busca pela atualidade da informação também pode ser representada por um fluxo constante (sem início ou fim definido) e nãolinear. Os decisores frequentemente estão procurando atualizar suas informações na tentativa de torná-las relevantes para a tarefa ou decisão em questão, processo considerado importante por diversos autores, como Mintzberg, Raisinghani e Théorêt (1976), Nutt (1984), Clemen (1996), entre outros.

Para ilustrar essa dimensão, Moody e Walsh (1999) analisaram a informação como um ativo organizacional e definiram seu comportamento como um bem econômico que é perecível. A informação "perde seu valor" à medida que o tempo passa.

A atualidade está também intimamente ligada à construção da identidade e à representação de ambientes sensíveis. Com base no entendimento compartilhado do contexto e da integralidade das informações, surge a necessidade da atualização das informações para a decisão.

\section{f) Focado em pistas e por pistas}

Na concepção de Weick (1995) as pistas são simples estruturas similares que representam indícios a partir dos quais as pessoas desenvolveram noções sobre o que pode estar ocorrendo. Tais pistas fornecem pontos de referência, ou núcleos, a partir dos quais as ideias podem ser ligadas em redes de significados (CHOO, 2003). As análises dessas pistas dependem do contexto no qual estão inseridas. 
A dimensão da qualidade da informação analisada nesta propriedade refere-se à concisão, que pode ser entendida como a extensão pela qual a informação é condensadamente apresentada. Nesta dimensão, busca-se mostrar, nas informações, fatores muito relevantes para que os tomadores de decisões possam, a partir desses indícios, utilizar pontos de referência para guiar suas ações, compreender o ambiente e criar suas identidades.

Um caso típico para ilustrar essa dimensão são os sinais de mercado. Quando novos concorrentes ou produtos concorrentes estão para serem lançados, o mercado apresenta sinais fracos, médios ou fortes que funcionam como pistas para direcionar os tomadores de decisões. Essas pistas constituem pontos de referência, e dependendo da interpretação do decisor, alinhado ao contexto, tornam-se um importante insumo para a decisão.

g) Governado mais pela plausibilidade do que pela precisão

As pessoas se comportam pragmaticamente quando criam significados, priorizando a plausibilidade sobre a precisão, entendimento este compartilhado por Choo (2003). O critério da precisão é secundário nesta análise do sensemaking por uma variedade de razões pois, para Weick (1995), as pessoas necessitam distorcer e filtrar para separar o sinal útil do ruído - caso não queiram ser saturadas por enorme carga de informações. O sensemaking busca a plausibilidade, a coerência, a racionalidade e a instrumentalidade.

Relacionadas com esta propriedade estão as dimensões da consistência, da veracidade e da ausência de erros. Essas três dimensões buscam fornecer, aos tomadores de decisão, os indícios básicos e os pontos de referência para o ato decisório. As informações precisam ser verdadeiras e livres de erros - uma vez que estes podem corrompêlas com tendências - porém não necessitam ser excessivamente minuciosas. Para a tomada de decisões, as informações são sempre parciais e necessitam da interpretação para que se tornem úteis. Corrobora neste sentido a observação de Daft e Weick (2005), apontando que a interpretação é o processo de tradução desses eventos, de desenvolvimento de modelos para compreensão, de descoberta de significados e de montagem de esquemas conceituais por gestores-chaves.

O foco na complexidade do sensemaking humano permite entender o fenômeno por meio do significado que as pessoas atribuem a ele (Klein e Myers, 1999). Destarte, o processo de interpretação move-se a partir de um entendimento prévio das partes até o completo e global entendimento do contexto. Esse entendimento pode refletir uma mensuração da qualidade da informação mais completa, pois neste caso, esta abordagem permite levar em conta o contexto no qual a informação está sendo utilizada.

\section{REFLEXÕES}

Neste ensaio teórico, buscou-se ampliar a análise da qualidade da informação explorando-a sob a ótica do sensemaking. Esse termo, abordado por Weick (1995), foi utilizado em sua proposição original (sem ser traduzido) para que fosse mantido o contexto da criação de sentido proposta pelo autor.

Sabe-se que a escolha das dimensões da qualidade da informação é, primeiramente, baseada em um entendimento intuitivo, em uma experiência pessoal coletiva ou mesmo em uma revisão da literatura (WAND e WANG, 1996). Entretanto, os mesmos autores observam que a literatura mostra que não há um consenso sobre as dimensões da qualidade da informação. Partindo do entendimento que a criação de sentido é um ato social e também da afirmação de Daft e Weick (2005) - que a literatura organizacional sugere que as organizações e os indivíduos tomam decisões de várias maneiras - este ensaio apresenta uma ótica diferente para se avaliar a qualidade da informação utilizada na tomada de decisão.

Após a aproximação entre os conceitos do sensemaking e as dimensões apresentadas por Pipino , Lee e Yang (2002), reforça-se a premissa da subjetividade na "valoração" da informação utilizada e a busca por uma forma de se mensurar 
A qualidade da informação para a tomada de decisão sob a perspectiva do sensemaking: uma ampliação do campo

essa qualidade. Foi possível ainda compreender como importantes capacidades e habilidades humanas envolvidas na decisão estão presentes na composição da qualidade da informação. Somase a isto a importância na busca da plausibilidade e da compreensão sociocontextual, conforme concluíram Nehmy e Paim (1998) em seu artigo.

Como agenda de pesquisa, a partir deste ensaio, propõe-se a utilização desta abordagem em pesquisas empíricas, explorando de forma detalhada o dia a dia dos atores envolvidos no processo de decisão. A análise em profundidade da aplicação deste relacionamento poderia fornecer mais indícios sobre a qualidade da informação, auxiliando, dessa maneira, o relacionamento entre os fornecedores e os consumidores de informação e também a concepção de sistemas e orientação de investimentos tecnológicos nas organizações.

Artigo submetido em 26/06/2009 e aceito em 29/03/2011

\section{REFERÊNCIAS}

BAZERMAN, M. Processo decisório. Rio de Janeiro: Elsevier, 2004.

BEAL, A. Gestão estratégica da informação: como transformar a informação e a tecnologia da informação em fatores de crescimento e de alto desempenho nas organizações. São Paulo: Atlas, 2004.

CAPURRO, R. Toward an Information Ecology. In: WORMELL, I. (Ed.) Information and Quality. London: Taylor Graham 1990, 122-139.

CAPURRO, R.; HJORLAND, B. O conceito da informação. Perspectivas em Ciência da Informação. Belo Horizonte, v. 12, n. 1, p. 148-207, Jan/Abr, 2007.

CHECKLAND, P. Information, systems and information systems: making sense of the field. West Sussex: John Wiley \& Sons, 1998.

CIBORRA, C. Teams, markets and systems: business innovation and information technology. Cambridge: Cambridge University Press, 1992.

CLEMEN, R. T. Making hard decisions. 2. ed. Pacific Grove: Duxbury, 1996.

COHEN, M. D; MARCH, J. G.; OLSEN, J. P. A garbage can model of organizational choice. Administrative Science Quarterly. New York, v. 17, n. 1, p. 1-25, Mar. 1972.

CHOO, C. W. A organização do conbecimento: Como as organizações usam a informação para criar significado, construir conhecimento e tomar decisões. São Paulo: Editora Senac São Paulo, 2003.
DAFT, R. L.; WEICK, E. K. Por um modelo de organização concebido como sistema interpretativo. Revista de Administração de Empresas. v. 45, n. 4, p. 73-86, 2005.

DERVIN, B. An overview of Sense-Making research: Concepts, methods and results to date. In: International Communication Association annual meeting, 1983. Dallas, Texas, USA. Disponível em: < http:// faculty.washington.edu/wpratt/MEBI598/Methods/An\%20 Overview $\% 20$ of $\% 20$ Sense-Making\%20Research $\% 201983 \mathrm{a}$.htm> Acesso em 25 maio 2011.

Sense-making theory and practice: an overview of user interests in knowledge seeking and use. Journal of Knowledge Management, v. 2, n. 2, p. 36-46, Dec. 1998

EVEN, A.; SHANKARANARAYANAN, G. Utility-Driven Assessment of Data Quality. The DATA BASE for Advances in Information Systems. v. 38, n. 2, May, 2007.

EISENHARDT, K. M.; ZBARACKI, M. J. Strategic Decision Making. Strategic Management Journal. West Sussex, v. 13, p. 17-37, Feb. 1992.

HAMMOND, J. S.; KEENEY, R. L.; RAIFFA, H. Decisões inteligentes. Rio de Janeiro: Elsevier, 2004.

JUNG W., A review of research: an investigation of the impact of data quality on decision performance. In: International Symposium on Information \& Communication Technologies (ISITC'04), 2004.

KLEIN, H. K.; MYERS, M. D. A set of principles for condicting and evaluating interpretive field studies in information systems. MIS Quarterly. v. 23, n. 1, p. 67-93, March, 1999.

KUHLTHAU, C. C. Seeking meaning: a process approach to library and information services. $2^{\text {nd }}$. Ed., Westport : Libraries Unlimited, 2004.

LAUDON, K. C.; LAUDON, J. P. Management information systems. 4. ed. São Paulo: Prentice-Hall, Inc., 1996.

MARCH, J.G. e OLSEN, J. Ambiguity and choice in organizations. Bergen: Universitetsforlaget, 1976.

MASUDA, Y. A sociedade da informação como sociedade pós-industrial. Rio de Janeiro: Editora Rio. 1982.

McGEE, J V.; PRUSAK, L. Gerenciamento estratégico da informação: aumente a competitividade e a eficiência de sua empresa utilizando a informação como uma ferramenta estratégica. 12. ed. Rio de Janeiro: Campus, 1994.

MINTZBERG, H; RAISINGHANI, D; THÉORÊT, A. The structure of 'unstructured' decision processes. Administrative Science Quarterly. New York, v. 21, n. 02, 246-275, June 1976.

MOODY, D.; WALSH, P. Measuring the value of information: an asset evaluation approach. In: European Conference on Information Systems, 1999. Disponível em: < http://wwwinfo.deis.unical.it/ zumpano/2004-2005/PSI/lezione2/ValueOfInformation.pdf>. Acesso em: 26 maio 2011.

NEHMY, R.M.Q. e PAIM, I. A desconstrução do conceito de "qualidade da informação". Ciência da Informação. v. 27, n. 1, p. 36-45, Jan/Abr., 1998. 


\section{Gustavo Abib}

NUTT, P. C. Types of organizational decision process. Administrative Science Quarterly. New York, v. 29, p. 414-450, Sept. 1984.

OLETO, R. R. Percepção da qualidade da informação. Ciência da Informação. v. 35, n. 1, p. 57-62, Jan/Abr., 2006.

PIPINO, L.L.; YANG, W. L. e WANG, R. Y. Data Quality Assessment. Communications of the ACM. v. 45, n. 4, p. 211-218, April, 2002.

REDMAN, T.C. Data quality for the information age. Boston: Artech House, 1996.

STARBUCK, W.H., e MILLIKIN, F.J. Executives perceptual filters: What they notice and how they make sense. In: HAMBRICK, D.C.; BRANDON, G.L. (Eds.), The executive effect: Concepts and methods for studying top managers. Greenwich: JAI Press, 1988.

WALSHAN, G. The Emergence of Interpretativism in IS Research. Information System Research. v. 6, n. 4, p. 376-394, December, 1995.
WAND, Y.; WANG, R. Y. Data Quality Dimensions in Ontological Foundations. Communications of the ACM. v. 39, n. 11, p. 86-95, November, 1996.

WANG, R.Y, STOREY, V.C. e FIRTH, C.P. A Framework for Analysis of Data Quality Research. IEEE Transactions of Knowledge and Data Engineering, v.7, n.4, p. 623-640, 1995.

WEICK, K. E. Sensemaking in organizations. Thousand Oaks: Sage Publications, 1995.

WEICK, K.; SUTCLIFFE, K.M.; OBSTFELD, D. Organizing and the process of sensemaking. Organization Science, n.16, v.4, p.409-421, 2005.

WILSON, D.; JARZABKOWSKI, P. Pensando e agindo estrategicamente: novos desafios para a análise estratégica. Revista de Administração de Empresas. São Paulo, v. 44, n. 4, p.11-20, set. 2004.

WILSON T.D. Human Information Behavior. Special Issue on Information Science Research. v. 3, n. 